\title{
BMJ Open Psychiatric disorders as risk factors for the development of type 2 diabetes mellitus: an umbrella review protocol
}

\author{
Nanna Lindekilde, ${ }^{\oplus 1}$ Giesje Nefs, ${ }^{2,3}$ Jan Erik Henriksen, ${ }^{4}$ Mathias Lasgaard,,${ }^{1,5}$ \\ Miranda Schram, ${ }^{6}$ Katrine Rubin, ${ }^{7}$ Femke Rutters, ${ }^{8}$ Mika Kivimaki, ${ }^{9}$ Frans Pouwer ${ }^{1,4}$
}

To cite: Lindekilde N, Nefs G, Henriksen JE, et al. Psychiatric disorders as risk factors for the development of type 2 diabetes mellitus: an umbrella review protocol. BMJ Open 2019;9:e024981. doi:10.1136/ bmjopen-2018-024981

- Prepublication history and additional material for this paper are available online. To view these files, please visit the journal online (http://dx.doi. org/10.1136/bmjopen-2018024981).

Received 25 June 2018 Revised 5 December 2018 Accepted 30 April 2019

Check for updates

(c) Author(s) (or their employer(s)) 2019. Re-use permitted under CC BY-NC. No commercial re-use. See rights and permissions. Published by BMJ.

For numbered affiliations see end of article.

Correspondence to

Nanna Lindekilde;

nlindekilde@health.sdu.dk

\section{ABSTRACT}

Introduction Numerous longitudinal studies, systematic reviews and meta-analyses have examined psychiatric disorders as risk factors for the development of type 2 diabetes mellitus. A more comprehensive overview of the area is warranted to summarise current evidence and discuss strengths and weaknesses to guide future research.

Aim The aim of this umbrella review is to determine whether and to what extent different psychiatric disorders are associated with the development of type 2 diabetes mellitus. Furthermore, the umbrella review also assesses the evidence on potential mediating mechanisms.

Methods and analysis The present umbrella review will consist of a comprehensive systematic search of published systematic reviews and meta-analyses of observational longitudinal studies investigating whether a psychiatric disorder is associated with the risk of developing type 2 diabetes. PubMed, Embase, PsychINFO and the Cochrane Database of Systematic Reviews will be searched, and the results will be screened for inclusion by two independent reviewers. Furthermore, the reference lists of included publications will be manually searched. Two independent reviewers will extract data and assess the methodological quality in the included systematic reviews and metaanalyses. Evidence on potential mediating mechanisms included in the systematic reviews and meta-analyses will also be reviewed. The implications of the overview will be discussed in light of the quality of the included studies, and suggestions for clinical practice and future research will be made.

Ethics and dissemination Ethical approval is not required for this umbrella review. Our review will be submitted for publication in a peer-reviewed international journal using open access option if available. The results will also be disseminated at international conferences. PROSPERO registration number CRD42018096362

\section{INTRODUCTION}

Type two diabetes mellitus is a chronic metabolic condition that is increasingly common worldwide. ${ }^{1}$ Nine in ten diabetes cases are diagnosed with type 2 diabetes. ${ }^{2}$ Several factors may contribute to the increase in the number of people with type 2 diabetes, including the ageing of populations, ${ }^{3}$ an

\section{Strengths and limitations of this study}

To our knowledge, this is the first protocol for an umbrella review on psychiatric disorders as risk factors for type 2 diabetes.

- A broad search strategy will be used to ensure a comprehensive synthesis of existing systematic reviews and meta-analyses in this area.

- The methodological quality of the included systematic reviews and meta-analyses will be assessed.

- Assessment of data extraction and methodological quality will be performed by two independent reviewers.

- As in other umbrella reviews, the quality of the summary findings depends on the quality and content of the systematic reviews and meta-analyses that are available.

increased number of people with a sedentary lifestyle, ${ }^{3}$ the global obesity epidemic, ${ }^{34}$ and the increase in life expectancy. ${ }^{5}$ Furthermore, earlier and improved recognition of type 2 diabetes has increased in the number of people with diagnosed type 2 diabetes. ${ }^{3}$

A recent narrative review summarised evidence on different mental health problems (including psychiatric diseases, but also forms of chronic stress) as risk factors for type 2 diabetes. ${ }^{6}$ The review concluded that longitudinal studies support an association of depression, anxiety, sleeping problems, 'general emotional stress', 'anger' and 'hostility' with increased risk of type 2 diabetes. Findings were mixed regarding 'childhood neglect', 'life events' and 'work stress'. ${ }^{6}$ More recently, de Jonge and colleagues studied cross-sectional associations between 16 different Diagnostic and Statistical Manual of Mental Disorders (DSM)-IV psychiatric disorders and diabetes mellitus in over 52000 adults. ${ }^{7}$ Depression, intermittent explosive disorder, binge eating disorder and bulimia nervosa were associated with increased risk of diabetes mellitus after adjustment for comorbidity. ${ }^{7}$ The type of diabetes was unknown and no temporal 
inferences could be made due to the cross-sectional design. $^{7}$

Several systematic reviews and meta-analyses have also investigated whether specific psychiatric disorders are associated with the risk of developing type 2 diabetes. ${ }^{8-13}$ Mezuk $e$ al, for example, included 13 prospective studies and found that depression was associated with a 1.6-fold increased risk of developing type 2 diabetes during a follow-up of 3.0-15.6 years. ${ }^{8}$ Based on 12 longitudinal studies, Smith et al found that people with a diagnosed anxiety disorder or elevated anxiety symptoms had a 1.3-fold increased risk of developing type 2 diabetes. ${ }^{9}$ Stubbs et al investigated 25 studies and concluded that people with schizophrenia had almost double the risk of developing type 2 diabetes compared with those without that psychiatric disorder. ${ }^{10}$ Furthermore, in two other meta-analyses of four and five studies, Vancampfort and colleagues found that people with bipolar disorder or post-traumatic stress disorder had a 1.5-fold increased risk of type 2 diabetes. ${ }^{11}{ }^{12} \mathrm{~A}$ further meta-analysis on different eating disorders found bulimia nervosa being a risk factor for type 2 diabetes, whereas people with anorexia nervosa had a reduced risk of developing type 2 diabetes, and the association of binge eating disorder with type 2 diabetes was not statistically significant. ${ }^{13}$ However, an important limitation was the small number of included studies. ${ }^{13}$

Different psychiatric disorders may increase the risk of the development of type 2 diabetes through several pathways involving both behavioural and physiological mechanisms, including, for example, less healthy eating behaviours in terms of quality and quantity of food, physical inactivity, smoking and higher alcohol consumption. ${ }^{14}$ These factors, in turn, are associated with an increased risk of type 2 diabetes. ${ }^{15}$ Furthermore, the pharmacological treatment of various psychiatric disorders may affect type 2 diabetes risk. ${ }^{16}{ }^{17}$ For example, the association between antipsychotic drug use and development of type 2 diabetes is suggested to be attributable to weight gain implicated by hormones, neurotransmitters and neuropeptides that regulate appetite stimulation and consequently increase food consumption (eg, leptin, histamine H1 receptor and melanocortin 4 receptor) ${ }^{16}$ Weight-independent insulin resistance and insulin secretion may also contribute to the association. ${ }^{16}$ Further potential physiological mechanisms are hypercortisolism, resulting from long-term activation of the hypothalamic-pituitaryadrenal axis, ${ }^{18}$ changes in the immune function ${ }^{19}$ and inflammatory markers, such as elevated levels of interleukin- 6 and $\mathrm{C}$ reactive protein, ${ }^{20}$ altered levels of specific cytokines and chemokines ${ }^{21}$ and changes in the number and activation state of various leucocyte populations, ${ }^{21}$ all of which might impair glucose metabolism.

In sum, the number of systematic reviews and meta-analyses is increasing but has applied different methodologies and thus has not led to a coherent overview on psychiatric disorders as risk factors for type 2 diabetes. A novel method for a higher level synthesis of evidence is the so-called 'umbrella review', also known as 'review of reviews'. An umbrella review contributes to a comprehensive overview and aims to compile all the evidence in a specific area. These field synopses have the ability to clarify what is still unknown and require future research. ${ }^{22}{ }^{23}$ Furthermore, umbrella reviews typically include a methodological assessment of the included systematic reviews and meta-analyses, which contributes to a quality assessment of the existing evidence. ${ }^{22}{ }^{23}$ Obviously, the findings of umbrella reviews are dependent on the quality of the included systematic reviews and meta-analyses, which is an important methodological limitation. ${ }^{22} 23$

A recent umbrella review by Bellou and colleagues was the first of its kind to summarise risk factors for the development of type 2 diabetes. ${ }^{24} \mathrm{~A}$ broad range of risk factors was explored, including specific biomarkers, dietary factors, lifestyle and environmental factors, as well as medical history, including psychiatric disorders. Although the umbrella review by Bellou and colleagues had a broad and general focus, it did not discuss psychiatric disorders or potential pathways in detail. A comprehensive umbrella review with focus on specific psychiatric disorders and potential pathways is therefore needed for a synthesis of the numerous systematic reviews and meta-analyses in this area. By clarifying the strengths and weaknesses of the existing evidence, an umbrella review will contribute to a more thorough understanding of the associations and potential underlying pathways and enhance the needs for future research. Our umbrella review will emphasise the methodological quality assessment of already conducted systematic reviews and meta-analyses.

\section{OBJECTIVE}

The aim of this umbrella review is to determine to what extent people with a psychiatric disorder have an increased risk of developing type 2 diabetes mellitus (compared with a reference group without psychiatric disorder). Furthermore, the umbrella review also aims to review evidence on mediating mechanisms.

\section{METHODS AND ANALYSIS}

This protocol was developed according to the Preferred Reporting Items for Systematic Reviews and Meta-Analyses Protocols (PRISMA-P) guidelines (see online supplementary file 1). In accordance with these guidelines, the present umbrella review protocol has been registered in the International Prospective Register of Systematic Reviews. Protocol amendments will be documented in PROSPERO. The procedure of the planned umbrella review is shown in figure 1 .

\section{Patient and public involvement}

The patients or the public have not been involved in developing the present protocol nor will they be involved in conducting the umbrella review. 
Systematic search in electronic databases for systematic reviews and meta-analyses

\section{Removal of duplicates}

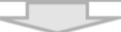

Screening in title/abstract for potential eligible systematic reviews and meta-analyses

Screening of full-text articles for eligible systematic reviews and meta-analyses

ren

Search of reference lists from eligible systematic reviews and meta-analyses

$$
\text { ए L }
$$

Extract data from the systematic reviews and metaanalyses

Assessment of methodological quality

$\sqrt{2}$

\section{Data summary and discussion}

Figure 1 Flowchart of the umbrella review.

\section{Search strategy}

\section{Database search}

The following four electronic databases will be searched: PubMed (1966-present), Embase (1974-present), PsychINFO (1967-present) and the Cochrane Database of Systematic Reviews (1992-present). Furthermore, a manual screening of the reference lists of the included publications will be completed.

\section{Search terms}

In the four electronic databases, we will conduct a systematic search using search words and Medical Subject Headings $(\mathrm{MeSH})$ terms combined using the Boolean logic operators (OR and AND). The search strategy will be based on three overall domains: psychiatric disorder, diabetes and systematic review/meta-analysis. Specific search words and MeSH terms within each domain will be combined using OR terms, and the domains will be combined by using AND terms. The domain of psychiatric disorders is coved by a comprehensive search string including terms and categories from the DSM-III or DSM-IV-TR (axis I and axis II) ${ }^{25} 26$ and the International Classification of Diseases, 10th Revision (ICD10), Classification of Mental Disorders (F00-F99). ${ }^{27}$ The search strategy has been generated in collaboration with a librarian and initially targeted PubMed, and will subsequently be adapted for the other databases. Date and language restrictions will not be used in the search strategy. Online supplementary file 2 shows the complete search string.

\section{Eligibility criteria}

Study design

The present umbrella review will include systematic reviews and meta-analyses of observational longitudinal studies in adult humans ( $\geq 18$ years).

\section{Inclusion and exclusion criteria}

We set the following inclusion and exclusion criteria:

- The study is a full systematic review with or without a meta-analysis, describing both a systematic search string and eligibility criteria.

- The papers that will be included in the systematic review/meta-analysis describe observational longitudinal studies in adult humans ( $\geq 18$ years) and include results based on longitudinal data.

- Type 2 diabetes and diabetes in general is included as an outcome determined, for example, by a diagnosis, medical reports, medical prescription or self-reported measurement (It is estimated that more than $90 \%$ of cases with diabetes mellitus have type 2 diabetes. $^{2}$ Therefore, we will include adult samples with 'diabetes in general, where no information is available on subtypes of diabetes' as we expect that the majority of the sample will actually have type 2 diabetes. We are aware of potential bias and will discuss this accordingly). However, papers reporting only on type 1 diabetes, latent autoimmune diabetes in adults or maturity-onset diabetes of the young and other specific types of diabetes except type 2 diabetes will be excluded.

- Distinct psychiatric disorders are studied as a risk factor for type 2 diabetes. The presence of a psychiatric disorder at baseline can be determined, for example, by a diagnostic interview (ICD or DSM classifications), data from medical charts, registries, medication prescription as a proxy measurement or by self-reported psychiatric diagnoses or elevated levels of clusters of psychiatric symptoms (eg, questionnaires measuring psychiatric disorders).

- Inclusion of a group with psychiatric disorder and a control group without a psychiatric disorder at baseline, all without having type two diabetes at baseline.

\section{Study selection}

The study selection will be handled in EndNote and in the software programme Covidence; in 2015, Covidence was selected by Cochrane to become the standard production platform for Cochrane Reviews. ${ }^{28}$ The initial search and the removal of duplicates will be performed by the first author. In all the identified publications, a screening of titles and abstracts for inclusion and exclusion criteria will be carried out by two independent 
reviewers. If eligibility of the publication is unsure, the publication will be identified as potentially relevant and will be enrolled in the next selection step. All publications identified as potentially relevant by at least one reviewer will be retrieved in full text and assessed for eligibility by two independent reviewers. In case of disagreement, the two reviewers will discuss this and, if necessary, a third reviewer will be included. Finally, the reference lists of the included publications are manually screened to identify publications, which fulfil the inclusion criteria. If both an original version and an updated version on a systematic review are identified, both papers will be included and discussed in the umbrella review. In the final umbrella review, a PRISMA flow diagram will show the selection flow and the number of included publications.

\section{Data extraction}

The included systematic reviews and meta-analyses will be independently reviewed by two reviewers, and information will be extracted using a predesigned data extraction form. Any discrepancies will be discussed until consensus is achieved. If any included papers are published in a non-English language not spoken by the reviewers, Google Translate will be used and a translator will be contacted if needed. Extracted information will include first author, year of publication, type of study, risk factor(s), type of assessment of risk factor(s), assessment of type 2 diabetes, the number of studies included, range of follow-up, total number of participants at baseline without diabetes mellitus, gender distribution and range of age of cases/controls. Furthermore, the description of and primary conclusions about potential mechanisms (eg, lifestyle behaviour and medication use) that link psychiatric disorders with diabetes risk will also be extracted. For meta-analyses, we will extract summary effect sizes (random effect size and/or fixed effect size, 95\% CI) and significance levels. When available, information of between-study heterogeneity (Cochrane $Q$ statistic or $\mathrm{I}^{2}$ and small-study effects will also be extracted.

\section{Assessment of methodological quality}

The methodological quality of each of the included systematic reviews and meta-analyses will be assessed independently by two reviewers using A MeaSurement Tool to Assess systematic Reviews, ${ }^{29}{ }^{30}$ which consists of an 11-item checklist. Disagreements will be resolved by discussion. The assessment of methodological quality will be included in the discussion of the findings from the respective systematic reviews and meta-analyses.

\section{DISCUSSION}

In the umbrella review, we will provide a high-level field synthesis and also discuss the results from the systematic reviews and meta-analyses for each psychiatric disorder as a potential risk factor for type 2 diabetes. Furthermore, mechanisms identified in the included systematic reviews and meta-analyses will be described and addressed. We will also focus on several methodological aspects, including the use of self-report versus diagnostic interview and the differences among psychiatric diagnoses and clusters of psychiatric symptoms. Furthermore, the characteristics of included primary studies in the systematic reviews and meta-analyses will be discussed. The results will be discussed in relation to the methodological quality assessment.

\section{ETHICS AND DISSEMINATION}

According to Danish law, formal ethical approval is not required for the present study, as primary data will not be collected. For the same reason, the project does not have to be reported to the Danish Data Protection Agency. The umbrella review will be submitted for publication in a peer-reviewed journal using open access option, if available, and results will also be disseminated at international conferences.

\section{Author affiliations}

${ }^{1}$ Department of Psychology, University of Southern Denmark, Odense, Denmark ${ }^{2}$ Diabeter, National Treatment and Research Center for Children, Adolescents and Young Adults with Type 1 Diabetes, Rotterdam, The Netherlands

${ }^{3}$ Department of Medical Psychology, Radboud University Medical Center, Radboud Institute for Health Sciences, Nijmegen, The Netherlands

${ }^{4}$ Steno Diabetes Centre Odense, Odense University Hospital, Odense, Denmark ${ }^{5}$ DEFACTUM Public Health \& Health Services Research, Central Denmark Region, Aarhus, Denmark

${ }^{6}$ Department of Internal Medicine, CARIM School for Cardiovascular Diseases, Maastricht University, Maastricht, The Netherlands

${ }^{7}$ Odense Patient Data Explorative Network (OPEN), Department of Clinical Research, University of Southern Denmark and Odense University Hospital, Odense, Denmark ${ }^{8}$ Department of Epidemiology and Biostatistics, Faculty of Medicine, Amsterdam UMC, Amsterdam, The Netherlands

${ }^{9}$ Department of Epidemiology and Public Health, University of College London, London, UK

Contributors FP is guarantor of the umbrella review. FP and NL had the original idea and designed the study with critical contributions from JEH and ML. NL wrote the first draft of the protocol and submitted the registration to PROSPERO. All authors (NL, GN, JEH, ML, MS, KHR, FR, MK and FP) have contributed to the manuscript and approved the final revised version of the manuscript that was resubmitted for publication.

Funding This work was supported by an unrestricted grant from the Faculty of Health Sciences, University of Southern Denmark, to FP. The financial provider is not involved in any other aspect of the project neither planning the design, data collection, analyses or interpretation of the results. MK is supported by the Medical Research Council (K013351, R024227 and S011676), NordForsk, Academy of Finland (311492) and a Helsinki Institute of Life Science fellowship.

Competing interests None declared.

Patient consent for publication Not required.

Provenance and peer review Not commissioned; externally peer reviewed.

Open access This is an open access article distributed in accordance with the Creative Commons Attribution Non Commercial (CC BY-NC 4.0) license, which permits others to distribute, remix, adapt, build upon this work non-commercially, and license their derivative works on different terms, provided the original work is properly cited, appropriate credit is given, any changes made indicated, and the use is non-commercial. See: http://creativecommons.org/licenses/by-nc/4.0/.

\section{REFERENCES}

1. International Diabetes Federation. IDF Diabetes Atlas. 8th ed. http:// www.diabetesatlas.org/.

2. American Diabetes Association. Diagnosis and classification of diabetes mellitus. Diabetes Care 2011;34(Suppl 1):60-71. 
3. Chatterjee S, Khunti K, Davies MJ. Type 2 diabetes. Lancet 2017;389:2239-51.

4. Smyth S, Heron A. Diabetes and obesity: the twin epidemics. Nat Med 2006;12:75-80.

5. Holden SE, Jenkins-Jones S, Morgan CL, et al. Prevalence, glucose control and relative survival of people with Type 2 diabetes in the UK from 1991 to 2013. Diabet Med 2017;34:770-80.

6. Pouwer F, Kupper N, Adriaanse MC. Does emotional stress cause type 2 diabetes mellitus? A review from the European Depression in Diabetes (EDID) Research Consortium. Discov Med 2010;9:112-8.

7. de Jonge P, Alonso J, Stein DJ, et al. Associations between DSM-IV mental disorders and diabetes mellitus: a role for impulse control disorders and depression. Diabetologia 2014;57:699-709.

8. Mezuk B, Eaton WW, Albrecht S, et al. Depression and type 2 diabetes over the lifespan: a meta-analysis. Diabetes Care 2008;31:2383-90.

9. Smith KJ, Béland M, Clyde M, et al. Association of diabetes with anxiety: a systematic review and meta-analysis. J Psychosom Res 2013;74:89-99.

10. Stubbs B, Vancampfort D, De Hert M, et al. The prevalence and predictors of type two diabetes mellitus in people with schizophrenia: a systematic review and comparative meta-analysis. Acta Psychiatr Scand 2015;132:144-57.

11. Vancampfort D, Mitchell AJ, De Hert M, et al. Prevalence and predictors of type 2 diabetes mellitus in people with bipolar disorder: a systematic review and meta-analysis. J Clin Psychiatry 2015;76:1490-9.

12. Vancampfort D, Rosenbaum S, Ward PB, et al. Type 2 diabetes among people with posttraumatic stress disorder: systematic review and meta-analysis. Psychosom Med 2016;78:465-73.

13. Nieto-Martínez R, González-Rivas JP, Medina-Inojosa JR, et al. Are Eating Disorders Risk Factors for Type 2 Diabetes? A Systematic Review and Meta-analysis. Curr Diab Rep 2017;17:138.

14. Scott $D$, Happell $B$. The high prevalence of poor physical health and unhealthy lifestyle behaviours in individuals with severe mental illness. Issues Ment Health Nurs 2011;32:589-97.

15. Wu Y, Ding Y, Tanaka $Y$, et al. Risk factors contributing to type 2 diabetes and recent advances in the treatment and prevention. Int $J$ Med Sci 2014;11:1185-200.
16. Whicher CA, Price HC, Holt RIG. Mechanisms in endocrinology: Antipsychotic medication and type 2 diabetes and impaired glucose regulation. Eur J Endocrinol 2018;178:R245-58.

17. Chen J, Huang XF, Shao R, et al. Molecular mechanisms of antipsychotic drug-induced diabetes. Front Neurosci 2017;11:643.

18. Stokes PE, Sikes CR. Hypothalamic-pituitary-adrenal axis in psychiatric disorders. Annu Rev Med 1991;42:519-31.

19. Jones KA, Thomsen $C$. The role of the innate immune system in psychiatric disorders. Mol Cell Neurosci 2013;53:52-62.

20. Wang X, Bao W, Liu J, et al. Inflammatory markers and risk of type 2 diabetes: a systematic review and meta-analysis. Diabetes Care 2013;36:166-75.

21. Donath MY, Shoelson SE. Type 2 diabetes as an inflammatory disease. Nat Rev Immunol 2011;11:98-107.

22. Aromataris E, Fernandez R, Godfrey CM, et al. Summarizing systematic reviews: methodological development, conduct and reporting of an umbrella review approach. Int J Evid Based Healthc 2015;13:132-40.

23. Biondi-Zoccai G. Umbrella Reviews. Evidence Synthesis with Overviews of Reviews and Meta- Epidemiologic Studies. Springer) 2016.

24. Bellou V, Belbasis L, Tzoulaki I, et al. Risk factors for type 2 diabetes mellitus: An exposure-wide umbrella review of meta-analyses. PLoS One 2018;13:e0194127.

25. American Psychiatric Association. Diagnostic and statistical manual of mental disorders: DSM-IV-TR. Washington, DC: American Psychiatric Association, 2000.

26. American Psychiatric Association. Diagnostic and statistical manual of mental disorders: DSM-III. Washington; DC: American Psychiatric Association: Author, 1980.

27. World Health Organization. The ICD-10 classification of mental and behavioural disorders: clinical descriptions and diagnostic guidelines. Geneva: World Health Organization, 2009.

28. Covidence [program]. Melbourne, Australia: Veritas Health Innovation. www.covidence.org (accessed 1 Jun 2018).

29. Shea BJ, Grimshaw JM, Wells GA, et al. Development of AMSTAR: a measurement tool to assess the methodological quality of systematic reviews. BMC Med Res Methodol 2007;7:10.

30. Shea BJ, Hamel C, Wells GA, et al. AMSTAR is a reliable and valid measurement tool to assess the methodological quality of systematic reviews. J Clin Epidemiol 2009;62:1013-20. 